\title{
Erratum: Origin of Orbital Ferromagnetism and Giant Magnetic Anisotropy at the Nanoscale [Phys. Rev. Lett. 96, 057206 (2006)]
}

\author{
A. Hernando, P. Crespo, and M. A. García \\ (Received 9 February 2006; published 3 March 2006)
}

DOI: 10.1103/PhysRevLett.96.089901

PACS numbers: 75.10. $-\mathrm{b}, 73.20 .-\mathrm{r}, 75.75 .+\mathrm{a}, 99.10 . \mathrm{Cd}$

References [1-4] in our Letter deal with the magnetism of some $\mathrm{C}$ based samples. As concerns proton irradiated graphite we must add in Ref. [4] two important references [1,2] omitted in the published version.

[1] P. Esquinazi et al., Phys. Rev. Lett. 91, 227201 (2003).

[2] K. Han et al., Adv. Mater. 15, 1719 (2003). 